\title{
Complete genome sequence of the thermophilic sulfur-reducer Hippea maritima type strain $\left(\mathrm{MH}_{2}{ }^{\mathrm{T}}\right)$
}

\author{
Marcel Huntemann ${ }^{1}$, Megan Lu, ${ }^{2,3}$, Matt Nolan², Alla Lapidus², Susan Lucas ${ }^{2}$, Nancy \\ Hammon $^{2}$, Shweta Deshpande ${ }^{2}$, Jan-Fang Cheng ${ }^{2}$, Roxanne Tapia ${ }^{2,3}$, Cliff Han ${ }^{2,3}$, Lynne \\ Goodwin ${ }^{2,3}$, Sam Pitluck ${ }^{2}$, Konstantinos Liolios ${ }^{2}$, Ioanna Pagani ${ }^{2}$, Natalia Ivanova ${ }^{2}$, Galina \\ Ovchinikova ${ }^{2}$, Amrita Pati ${ }^{2}$, Amy Chen ${ }^{1}$, Krishna Palaniappan ${ }^{1}$, Miriam Land ${ }^{2,4}$, Loren \\ Hauser $^{2,4}$, Cynthia D. Jeffries ${ }^{2,4}$, John C. Detter ${ }^{2,3}$, Evelyne-Marie Brambilla ${ }^{5}$, Manfred \\ Rohde $^{6}$, Stefan Spring ${ }^{5}$, Markus Göker ${ }^{5}$, Tanja Woyke ${ }^{2}$, James Bristow ${ }^{2}$, Jonathan A. Eisen ${ }^{2,7}$, \\ Victor Markowitz ${ }^{1}$, Philip Hugenholtz ${ }^{2,8}$, Nikos C. Kyrpides ${ }^{2}$, Hans-Peter Klenk ${ }^{5^{*}}$, and \\ Konstantinos Mavromatis ${ }^{2}$ \\ ${ }^{1}$ Biological Data Management and Technology Center, Lawrence Berkeley National \\ Laboratory, Berkeley, California, USA \\ ${ }^{2}$ DOE Joint Genome Institute, Walnut Creek, California, USA \\ ${ }^{3}$ Los Alamos National Laboratory, Bioscience Division, Los Alamos, New Mexico, USA \\ ${ }^{4}$ Oak Ridge National Laboratory, Oak Ridge, Tennessee, USA \\ ${ }^{5}$ DSMZ - German Collection of Microorganisms and Cell Cultures GmbH, Braunschweig, \\ Germany \\ ${ }^{6} \mathrm{HZI}$ - Helmholtz Centre for Infection Research, Braunschweig, Germany \\ ${ }^{7}$ University of California Davis Genome Center, Davis, California, USA \\ ${ }^{8}$ Australian Centre for Ecogenomics, School of Chemistry and Molecular Biosciences, The \\ University of Queensland, Brisbane, Australia \\ *Corresponding author: Hans-Peter Klenk
}

Keywords: anaerobic, motile, rod-shaped, Gram-negative, marine, moderately thermophilic, sulfur-reducer, Desulfurellaceae, GEBA

Hippea maritima (Miroshnichenko et al. 1999) is the type species of the genus Hippea, which belongs to the family Desulfurellaceae within the class Deltaproteobacteria. The anaerobic, moderately thermophilic marine sulfur-reducer was first isolated from shallow-water hot vents in Matipur Harbor, Papua New Guinea. H. maritima was of interest for genome sequencing because of its isolated phylogenetic location, as a distant next neighbor of the genus Desulfurella. Strain $\mathrm{MH}_{2}{ }^{\mathrm{T}}$ is the first type strain from the order Desulfurellales with a completely sequenced genome. The 1,694,430 bp long linear genome with its 1,723 proteincoding and 57 RNA genes consists of one circular chromosome and is a part of the Genomic Encyclopedia of Bacteria and Archaea project.

\section{Introduction}

Strain $\mathrm{MH}_{2}{ }^{\mathrm{T}}$ (DSM $10411=$ ATCC 700847) is the type strain of the species Hippea maritima, which is the type species of its genus Hippea [1]. The genus currently contains no other validly named species [2], but two other strains belonging to the species were isolated from shallow-water hot vents in New Zealand and Papua New Guinea [1]. The type strain was isolated during a cruise of the Russian scientific vessel $A$. Nesmeyanov through shallow-water hot vents of the south-western Pacific Ocean, environments that are typical for anaerobic, thermophilic, sulfur-reducing bacteria [1]. The genus is named after the German microbiologist Hans Hippe, in recognition of his significant contribution to the characterization of novel, obligately anaerobic prokaryotes and the understanding of their physiology. The species epithet is derived from the Latin word maritima (inhabiting marine environments) [2]. Here we present a summary classification and a set of features for $H$. maritima strain $\mathrm{MH}_{2}{ }^{\mathrm{T}}$, together with the description of the complete genomic sequencing and annotation. 


\section{Classification and features}

A representative genomic $16 \mathrm{~S}$ rRNA sequence of strain $\mathrm{MH}_{2}{ }^{\mathrm{T}}$ was compared using NCBI BLAST under default settings (e.g., considering only the high-scoring segment pairs (HSPs) from the best 250 hits) with the most recent release of the Greengenes database [3] and the relative frequencies, of taxa and keywords (reduced to their stem [4]) were determined, weighted by BLAST scores. The most frequently occurring genera were Desulfurella (38.7\%), Desulfovibrio (15.2\%), Deferribacter $(10.8 \%)$, Thermotoga (10.8\%) and Hippea (8.6\%) (44 hits in total). Regarding the single hit to sequences from members of the species, the average identity within HSPs was $99.9 \%$, whereas the average coverage by HSPs was $82.7 \%$. Among all other species, the one yielding the highest score was Desulfurella multipotens, which corresponded to an identity of $89.6 \%$ and an HSP coverage of $82.6 \%$. (Note that the Greengenes database uses the INSDC (= EMBL/NCBI/DDBJ) annotation, which is not an authoritative source for nomenclature or classification.) The highest-scoring environmental sequence was AF232926 ('United Kingdom: Montserrat geothermal springs clone MS10 proteobacterium'), which showed an identity of $88.9 \%$ and a HSP coverage of $73.0 \%$. The most frequently occurring keywords within the labels of environmental samples which yielded hits were 'microbi' (5.0\%), 'spring' (2.9\%), 'sediment' (2.4\%), 'soil' (2.3\%) and 'industri' (2.2\%) (206 hits in total). Environmental samples which yielded hits of a higher score than the highest scoring species were not found.

The 16S rRNA based tree in Figure 1 shows the phylogenetic neighborhood of $H$. maritima. The sequence of the two identical 16S rRNA genes differs by one nucleotide from the previously published 16S rRNA sequence (Y18292).

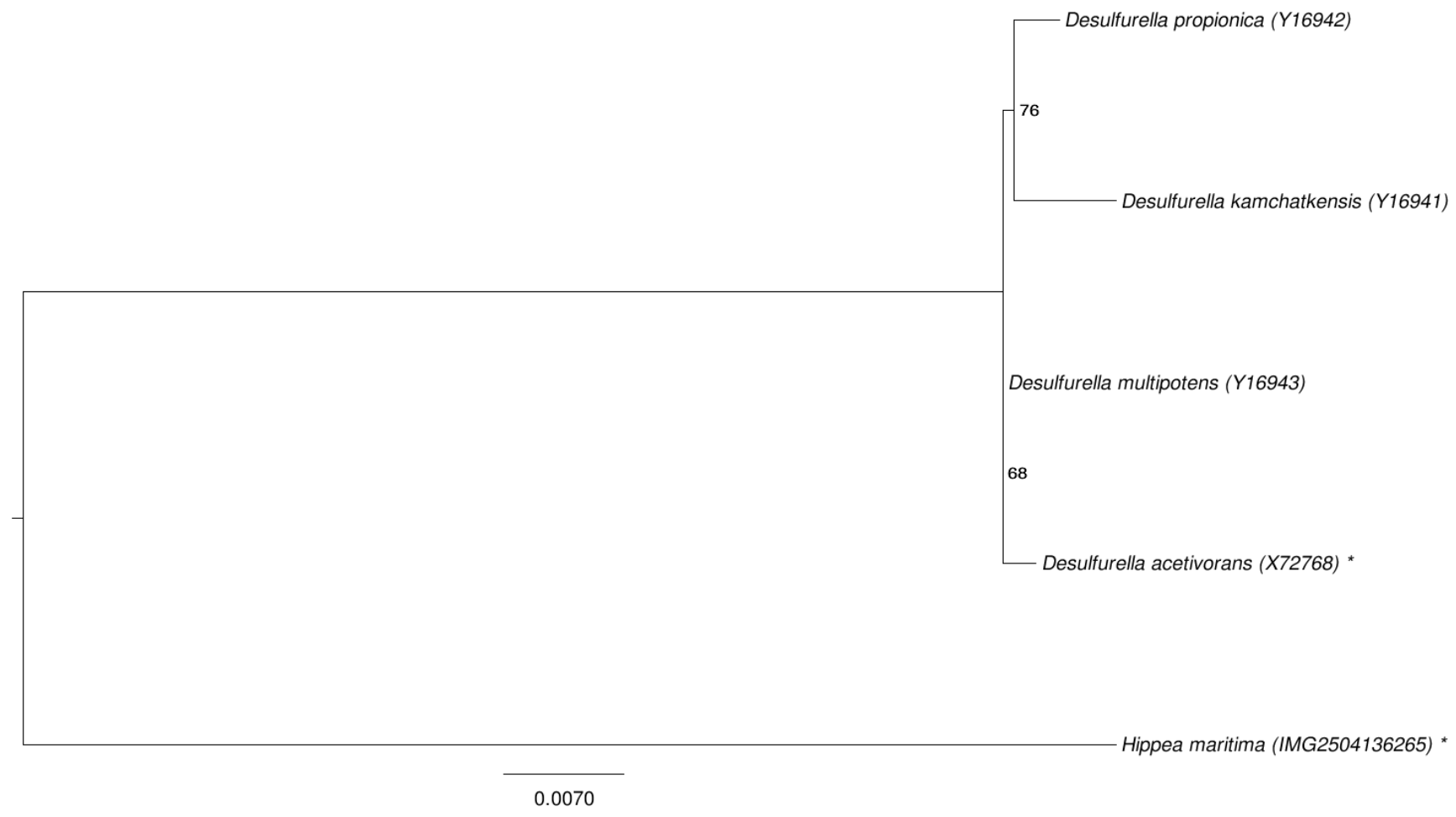

Figure 1. Phylogenetic tree highlighting the position of $H$. maritima relative to the other type strains within the family Desulfurellaceae. The tree was inferred from 1,526 aligned characters $[5,6]$ of the $16 \mathrm{~S}$ rRNA gene sequence under the maximum likelihood criterion [7] and rooted in accordance to the current taxonomy. The branches are scaled in terms of the expected number of substitutions per site. Numbers next to bifurcations are support values from 700 bootstrap replicates [8] if larger than 60\%. Lineages with type strain genome sequencing projects registered in GOLD [9] are shown with an asterisk, those also listed as 'Complete and Published' with two asterisks. 
The cells of $H$. maritima are short rods ranging from 1-3 x 0.4-0.8 $\mu \mathrm{m}$ (Figure 2 and Table 1) that occur singly or in pairs [1]. H. maritima is motile by one polar flagellum [1] (not visible in Figure 2). Colonies are whitish-gray with diameters up to 0.5 $\mathrm{mm}$ [1]. H. maritima cultures require $2.5-3 \% \mathrm{NaCl}$ and $0.02 \%(\mathrm{w} / \mathrm{v})$ yeast extract for growth [1]. The temperature range for growth is between $40^{\circ} \mathrm{C}$ and $65^{\circ} \mathrm{C}$, with an optimum at $52-54^{\circ} \mathrm{C}$ [1]. Growth was observed over a pH range of 5.7 to 6.5 with an optimum around 6.0 [1].

All $H$. maritima strains can grow on molecular hydrogen, acetate, and saturated fatty acids and require elemental sulfur as the only known electron acceptor [1]. Strain $\mathrm{MH}_{3}$, isolated from Matupi
Harbor, was the only $H$. maritima strain growing on ethanol in the presence of elemental sulfur [1]. Fumarate supported only weak growth for all three known strains [1], whereas formate, propionate, butyrate, pyruvate, lactate, succinate, glucose, starch, peptone, methanol did not support growth [1]. $\mathrm{CO}_{2}$ and $\mathrm{H}_{2} \mathrm{~S}$ were the only detected end products [1].

\section{Chemotaxonomy}

No chemotaxonomical data were reported in the initial description of the organism [1] nor elsewhere, subsequently.

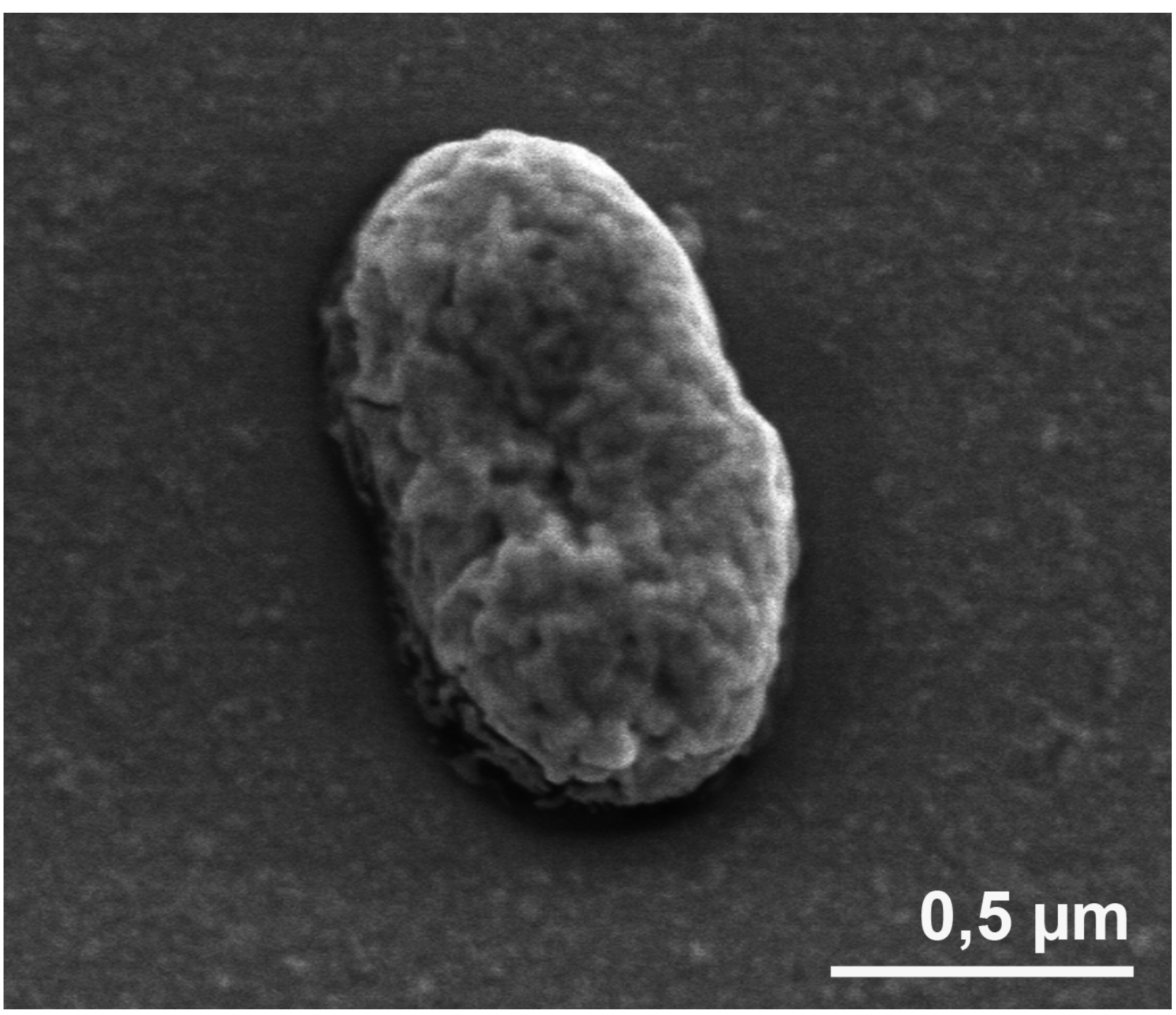

Figure 2. Scanning electron micrograph of H. maritima $\mathrm{MH}_{2}{ }^{\mathrm{T}}$ 
Table 1. Classification and general features of H. maritima $\mathrm{MH}_{2}{ }^{\top}$ according to the MIGS recommendations [10].

\begin{tabular}{|c|c|c|c|}
\hline MIGS ID & Property & Term & Evidence code \\
\hline & \multirow{8}{*}{ Current classification } & Domain Bacteria & TAS [11] \\
\hline & & Phylum Proteobacteria & TAS [12] \\
\hline & & Class Deltaproteobacteria & TAS $[13,14]$ \\
\hline & & Order Desulfurellales & TAS $[13,14]$ \\
\hline & & Family Desulfurellaceae & TAS $[14,15]$ \\
\hline & & Genus Hippea & TAS [1] \\
\hline & & Species Hippea maritima & TAS [1] \\
\hline & & Type strain $\mathrm{MH}_{2}$ & TAS [1] \\
\hline & Gram stain & negative & TAS [1] \\
\hline & Cell shape & short rods & TAS [1] \\
\hline & Motility & motile, one polar flagellum & TAS [1] \\
\hline & Sporulation & never observed & TAS [1] \\
\hline & Temperature range & $40-56^{\circ} \mathrm{C}$ & TAS [1] \\
\hline & Optimum temperature & $52-54^{\circ} \mathrm{C}$ & TAS [1] \\
\hline & Salinity & $2.5-3 \% \mathrm{NaCl}$ & TAS [1] \\
\hline \multirow[t]{3}{*}{ MIGS-22 } & Oxygen requirement & anaerobic & TAS [1] \\
\hline & Carbon source & saturated fatty acids (stearate, palmitate) & TAS [1] \\
\hline & Energy metabolism & $\begin{array}{l}\text { acetate, long-chain saturated fatty acids; } \\
\text { lithotrophic growth with } \mathrm{H}_{2} \text { and } \mathrm{S}_{0}\end{array}$ & TAS [1] \\
\hline MIGS-6 & Habitat & submarine hot vents & TAS [1] \\
\hline MIGS-15 & Biotic relationship & free-living & NAS \\
\hline \multirow[t]{3}{*}{ MIGS-14 } & Pathogenicity & none & NAS \\
\hline & Biosafety level & 1 & TAS [16] \\
\hline & Isolation & hot vents in tidal zone & TAS [1] \\
\hline MIGS-4 & Geographic location & Matupi Harbour, Papua New Guinea & TAS [1] \\
\hline MIGS-5 & Sample collection time & 1999 & TAS $[1,17]$ \\
\hline MIGS-4.1 & Latitude & -4.23 & NAS \\
\hline MIGS-4.2 & Longitude & 152.2 & NAS \\
\hline MIGS-4.3 & Depth & not reported & \\
\hline MIGS-4.4 & Altitude & approximately sea level & NAS \\
\hline
\end{tabular}

Evidence codes - IDA: Inferred from Direct Assay (first time in publication); TAS: Traceable Author Statement (i.e., a direct report exists in the literature); NAS: Non-traceable Author Statement (i.e., not directly observed for the living, isolated sample, but based on a generally accepted property for the species, or anecdotal evidence). These evidence codes are from of the Gene Ontology project [18]. If the evidence code is IDA, the property was directly observed by one of the authors or an expert mentioned in the acknowledgements.

\section{Genome sequencing and annotation Genome project history}

This organism was selected for sequencing on the basis of its phylogenetic position [19], and is part of the Genomic Encyclopedia of Bacteria and Archaea project [20]. The genome project is deposited in the Genomes On Line Database [9] and the complete genome sequence is deposited in GenBank. Sequencing, finishing and annotation were performed by the DOE Joint Genome Institute (JGI). A summary of the project information is shown in Table 2.

\section{Growth conditions and DNA isolation}

H. maritima $\mathrm{MH}_{2}{ }^{\mathrm{T}}$, DSM 10411, was grown anaerobically in medium 554 (HIPPEA medium) [21] at $55^{\circ} \mathrm{C}$. DNA was isolated from $0.5-1 \mathrm{~g}$ of cell paste using Jetflex Genomic DNA Purification Kit (GENOMED 600100) following the standard protocol as recommended by the manufacturer with the following modification to improve cell lysis: additional $20 \mu \mathrm{l}$ lysozyme $(100 \mathrm{mg} / \mu \mathrm{l})$ and $10 \mu \mathrm{l}$ mutalysin were used for $30 \mathrm{~min}$ incubation at $37^{\circ} \mathrm{C}$, followed by three hours incubation at $58^{\circ} \mathrm{C}$ with $20 \mu$ l proteinase $\mathrm{K}$. DNA is available through the DNA Bank Network [22]. 
Table 2. Genome sequencing project information

\begin{tabular}{lll}
\hline MIGS ID & Property & Term \\
\hline MIGS-31 & Finishing quality & Finished \\
MIGS-28 & Libraries used & $\begin{array}{l}\text { Three genomic libraries: one 454 pyrosequence standard library, } \\
\text { one 454 PE library (7.3 kb insert size), one Illumina library }\end{array}$ \\
MIGS-29 & Sequencing platforms & Illumina GAii, 454 GS FLX Titanium \\
MIGS-31.2 & Sequencing coverage & $1,213 \times$ Illumina; 29.6 × pyrosequence \\
MIGS-30 & Assemblers & Newbler version 2.3, Velvet version 0.7.63, phrap version SPS-4.24 \\
MIGS-32 & Gene calling method & Prodigal 1.4, GenePRIMP \\
& INSDC ID & CP002606 \\
& Genbank Date of Release & March 29, 2011 \\
& GOLD ID & Gc01705 \\
& NCBI project ID & 48195 \\
& Database: IMG-GEBA & 2504136000 \\
& Source material identifier & DSM 10411 \\
& Project relevance & Tree of Life, GEBA \\
\hline
\end{tabular}

\section{Genome sequencing and assembly}

The genome was sequenced using a combination of Illumina and 454 sequencing platforms. All general aspects of library construction and sequencing can be found at the JGI website [23]. Pyrosequencing reads were assembled using the Newbler assembler (Roche). The initial Newbler assembly, consisting of 70 contigs in one scaffold, was converted into a phrap [24] assembly by making fake reads from the consensus to collect the read pairs in the 454 paired end library. Illumina GAii sequencing data $(4,403.8 \mathrm{Mb})$ was assembled with Velvet [25] and the consensus sequences were shredded into $1.5 \mathrm{~kb}$ overlapped fake reads and assembled together with the 454 data. The 454 draft assembly was based on $66.2 \mathrm{Mb} 454 \mathrm{draft}$ data and all of the 454 paired end data. Newbler parameters are -consed a 50 -l 350 -g -m -ml 20. The Phred/Phrap/Consed software package [24] was used for sequence assembly and quality assessment in the subsequent finishing process. After the shotgun stage, reads were assembled with parallel phrap (High Performance Software, LLC). Possible mis-assemblies were corrected with gapResolution [23], Dupfinisher [26], or sequencing cloned bridging PCR fragments with subcloning or transposon bombing (Epicentre Biotechnologies, Madison, WI). Gaps between contigs were closed by editing in Consed, by PCR and by Bubble PCR primer walks (J.-F. Chang, unpublished). A total of 357 additional reactions and one shatter library were necessary to close gaps and to raise the quality of the finished sequence. Illumina reads were also used to correct potential base errors and increase consensus quality using a software Polisher developed at JGI [27].
The error rate of the completed genome sequence is less than 1 in 100,000. Together, the combination of the Illumina and 454 sequencing platforms provided $1,241.6 \times$ coverage of the genome. The final assembly contained 112,403 pyrosequence and $57,283,044$ Illumina reads.

\section{Genome annotation}

Genes were identified using Prodigal [28] as part of the Oak Ridge National Laboratory genome annotation pipeline, followed by a round of manual curation using the JGI GenePRIMP pipeline [29]. The predicted CDSs were translated and used to search the National Center for Biotechnology Information (NCBI) non-redundant database, UniProt, TIGR-Fam, Pfam, PRIAM, KEGG, COG, and InterPro databases. Additional gene prediction analysis and functional annotation were performed within the Integrated Microbial Genomes - Expert Review (IMG-ER) platform [30].

\section{Genome properties}

The genome consists of a 1,694,430 bp long linear chromosome with a $\mathrm{G}+\mathrm{C}$ content of $37.5 \%$ (Table 3 and Figure 3). Of the 1,780 genes predicted, 1,723 were protein-coding genes, and 57 RNAs; 46 pseudogenes were also identified. The majority of the protein-coding genes (76.4\%) were assigned with a putative function while the remaining ones were annotated as hypothetical proteins. The distribution of genes into COGs functional categories is presented in Table 4. 
Table 3. Genome Statistics

\begin{tabular}{lrr}
\hline Attribute & Value & \% of Total \\
\hline Genome size (bp) & $1,694,430$ & $100.00 \%$ \\
DNA coding region (bp) & $1,580,424$ & $93.27 \%$ \\
DNA G+C content (bp) & 634,975 & $37.47 \%$ \\
Number of replicons & 1 & \\
Extrachromosomal elements & 0 & \\
Total genes & 1,780 & $100.00 \%$ \\
RNA genes & 57 & $3.20 \%$ \\
rRNA operons & 2 & \\
Protein-coding genes & 1,723 & $96.80 \%$ \\
Pseudo genes & 46 & $2.58 \%$ \\
Genes with function prediction & 1,360 & $76.40 \%$ \\
Genes in paralog clusters & 182 & $10.22 \%$ \\
Genes assigned to COGs & 1,414 & $79.44 \%$ \\
Genes assigned Pfam domains & 1,485 & $83.43 \%$ \\
Genes with signal peptides & 261 & $14.66 \%$ \\
Genes with transmembrane helices & 423 & $23.76 \%$ \\
CRISPR repeats & 0 & \\
\hline
\end{tabular}

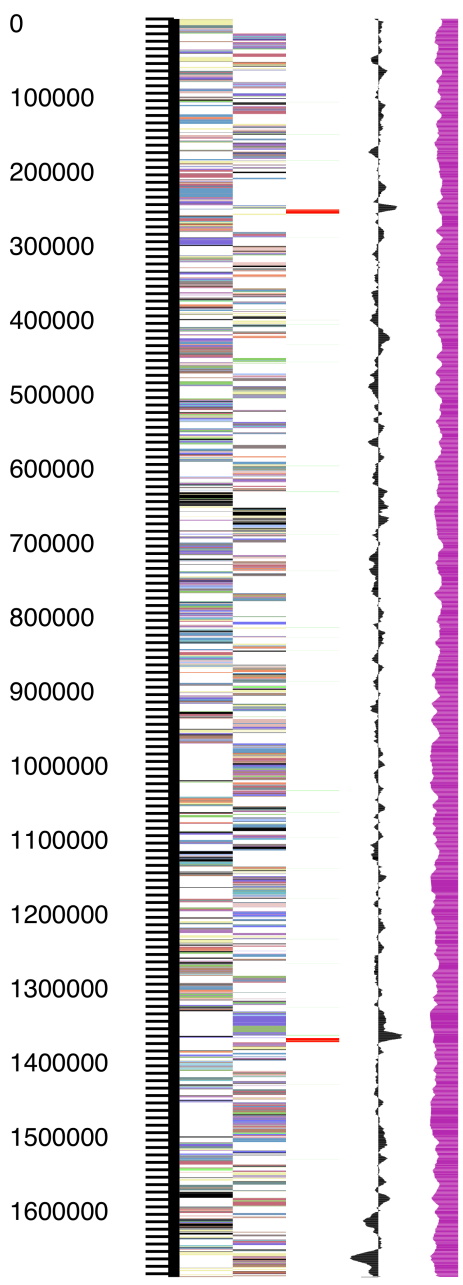

Figure 3. Graphical map of the linear chromosome. From left to right: Genes on forward strand (color by COG categories), Genes on reverse strand (color by COG categories), RNA genes (tRNAs green, rRNAs red, other RNAs black), GC content, GC skew. 
Table 4. Number of genes associated with the general COG functional categories

\begin{tabular}{crrl}
\hline Code & value & \% age & Description \\
\hline J & 133 & 8.5 & Translation, ribosomal structure and biogenesis \\
A & 0 & 0.0 & RNA processing and modification \\
K & 45 & 2.9 & Transcription \\
L & 119 & 7.6 & Replication, recombination and repair \\
B & 0 & 0.0 & Chromatin structure and dynamics \\
D & 19 & 1.2 & Cell cycle control, cell division, chromosome partitioning \\
Y & 0 & 0.0 & Nuclear structure \\
V & 11 & 0.7 & Defense mechanisms \\
T & 78 & 5.0 & Signal transduction mechanisms \\
M & 110 & 7.1 & Cell wall/membrane/envelope biogenesis \\
N & 69 & 4.4 & Cell motility \\
Z & 0 & 0.0 & Cytoskeleton \\
W & 0 & 0.0 & Extracellular structures \\
U & 59 & 3.8 & Intracellular trafficking, secretion, and vesicular transport \\
O & 68 & 4.4 & Posttranslational modification, protein turnover, chaperones \\
C & 107 & 6.9 & Energy production and conversion \\
G & 62 & 4.0 & Carbohydrate transport and metabolism \\
E & 147 & 9.4 & Amino acid transport and metabolism \\
F & 46 & 3.0 & Nucleotide transport and metabolism \\
H & 108 & 6.9 & Coenzyme transport and metabolism \\
I & 52 & 3.3 & Lipid transport and metabolism \\
P & 66 & 4.2 & Inorganic ion transport and metabolism \\
Q & 22 & 1.4 & Secondary metabolites biosynthesis, transport and catabolism \\
R & 146 & 9.4 & General function prediction only \\
S & 93 & 6.0 & Function unknown \\
- & 366 & 20.6 & Not in COGs \\
\hline & & & \\
\hline
\end{tabular}

\section{Acknowledgements}

We would like to gratefully acknowledge the help of Maren Schröder (DSMZ) for growing H. maritima cultures. This work was performed under the auspices of the US Department of Energy Office of Science, Biological and Environmental Research Program, and by the University of California, Lawrence Berkeley National Laboratory under contract No. DE-AC02-05CH11231,

\section{References}

1. Miroshnichenko ML, Rainey FA, Rohde M. Bonch-Osmolovskaya. Hippea maritima gen. nov, sp. nov., a new genus of thermophilic, sulphurreducing bacterium from submarine hot vents. Int I Syst Bacteriol 1999; 49:1033-1038. PubMed doi:10.1099/00207713-49-3-1033

2. Euzéby JP. List of bacterial names with standing in nomenclature: A folder available on the Internet.
Lawrence Livermore National Laboratory under Contract No. DE-AC52-07NA27344, and Los Alamos National Laboratory under contract No. DE-AC0206NA25396, UT-Battelle and Oak Ridge National Laboratory under contract DE-AC05-000R22725, as well as German Research Foundation (DFG) INST 599/1-2.

Int / Syst Bacterio/ 1997; 47:590-592. PubMed doi:10.1099/00207713-47-2-590

3. DeSantis TZ, Hugenholtz P, Larsen N, Rojas M, Brodie EL, Keller K, Huber T, Dalevi D, Hu P, Andersen GL. Greengenes, a Chimera-Checked $16 \mathrm{~S}$ rRNA Gene Database and Workbench Compatible with ARB. Appl Environ Microbiol 2006; 
72:5069-5072. PubMed

doi:10.1128/AEM.03006-05

4. Porter MF. An algorithm for suffix stripping. Program: electronic library and information systems 1980; 14:130-137.

5. Lee C, Grasso C, Sharlow MF. Multiple sequence alignment using partial order graphs. Bioinformatics 2002; 18:452-464. PubMed doi:10.1093/bioinformatics/18.3.452

6. Castresana J. Selection of conserved blocks from multiple alignments for their use in phylogenetic analysis. Mol Biol Evol 2000; 17:540-552. PubMed

7. Stamatakis A, Hoover P, Rougemont J. A rapid bootstrap algorithm for the RAxML Web servers. Syst Biol 2008; 57:758-771. PubMed doi:10.1080/10635150802429642

8. Pattengale ND, Alipour M, Bininda-Emonds ORP, Moret BME, Stamatakis A. How many bootstrap replicates are necessary? Lect Notes Comput Sci 2009; 5541:184-200. doi:10.1007/978-3-64202008-7_13

9. Liolios K, Chen IM, Mavromatis K, Tavernarakis N, Hugenholtz P, Markowitz VM, Kyrpides NC. The Genomes On Line Database (GOLD) in 2009: status of genomic and metagenomic projects and their associated metadata. Nucleic Acids Res 2010; 38:D346-D354. PubMed doi:10.1093/nar/gkp848

10. Field D, Garrity G, Gray T, Morrison N, Selengut J, Sterk P, Tatusova T, Thomson N, Allen MJ, Angiuoli SV, et al. The minimum information about a genome sequence (MIGS) specification. Nat Biotechnol 2008; 26:541-547. PubMed doi:10.1038/nbt1360

11. Woese CR, Kandler O, Wheelis ML. Towards a natural system of organisms: proposal for the domains Archaea, Bacteria, and Eucarya. Proc Natl Acad Sci USA 1990; 87:4576-4579. PubMed doi:10.1073/pnas.87.12.4576

12. Garrity GM, Bell JA, Lilburn T. Phylum XIV. Proteobacteria phyl. nov. In: Brenner DJ, Krieg NR, Staley JT, Garrity GM (eds), Bergey's Manual of Systematic Bacteriology, second edition, vol. 2 (The Proteobacteria), part B (The Gammaproteobacteria), Springer, New York, 2005, p. 1.

13. Kuever J, Rainey FA, Widdel F. Class VI. Deltaproteobacteria class nov. In: Brenner DJ, Krieg NR, Staley JT Garrity GM (eds), Bergey's Manual of Systematic Bacteriology, second edition, vol. 2 (The Proteobacteria), part C (The Alpha-, Beta-,
Delta-, and Epsilonproteobacteria), Springer, New York, 2005, p. 922

14. Validation list No. 107. Int / Syst Evol Microbiol 2006; 56:1-6. PubMed doi:10.1099/ijs.0.64188-0

15. Kuever J, Rainey FA, Widdel F. Family I. Desulfurellaceae fam. nov. In: Brenner DJ, Krieg NR, Staley JT Garrity GM (eds), Bergey's Manual of Systematic Bacteriology, second edition, vol. 2 (The Proteobacteria), part C (The Alpha-, Beta-, Delta-, and Epsilonproteobacteria), Springer, New York, 2005, p. 923.

16. BAuA. Classification of bacteria and archaea in risk groups. TRBA 2005; 466:205.

17. Miroshnichenko ML, Gongadze GM, Rainey FA, Kostyukova AS, Lysenko AM, Chernyhl NA, Bonch-Osmolovskaya EA. Thermococcus gorgonarius sp. nov. and Thermococcus pacificus sp. nov.: heterotrophic extremely thermophilic archaea from New Zealand submarine hot vents. Int J Syst Bacteriol 1998; 48:23-29. PubMed doi:10.1099/00207713-48-1-23

18. Ashburner M, Ball CA, Blake JA, Botstein D, Butler $\mathrm{H}$, Cherry JM, Davis AP, Dolinski K, Dwight SS, Eppig JT, et al. Gene Ontology: tool for the unification of biology. Nat Genet 2000; 25:25-29. $\underline{\text { PubMed doi:10.1038/75556 }}$

19. Klenk HP, Göker M. En route to a genome-based classification of Archaea and Bacteria? Syst Appl Microbiol 2010; 33:175-182. PubMed doi:10.1016/j.syapm.2010.03.003

20. Wu D, Hugenholtz P, Mavromatis K, Pukall R, Dalin E, Ivanova NN, Kunin V, Goodwin L, Wu $M$, Tindall BJ, et al. A phylogeny-driven genomic encyclopaedia of Bacteria and Archaea. Nature 2009; 462:1056-1060. PubMed doi:10.1038/nature08656

21. List of growth media used at DSMZ: http://www.dsmz.de/microorganisms/media_list.p hp.

22. Gemeinholzer B, Dröge G, Zetzsche H, Haszprunar G, Klenk HP, Güntsch A, Berendsohn WG, Wägele JW. The DNA Bank Network: the start from a German initiative. Biopreservation and Biobanking 2011; 9:51-55. doi:10.1089/bio.2010.0029

23. The DOE Joint Genome Institute. http://www.jgi.doe.gov

24. Phrap and Phred for Windows. MacOS, Linux, and Unix. http://www.phrap.com 
25. Zerbino DR, Birney E. Velvet: algorithms for de novo short read assembly using de Bruijn graphs. Genome Res 2008; 18:821-829. PubMed doi:10.1101/gr.074492.107

26. Han C, Chain P. Finishing repeat regions automatically with Dupfinisher. in Proceeding of the 2006 international conference on bioinformatics \& computational biology. Arabnia HR, Valafar $\mathrm{H}$ (eds), CSREA Press. June 26-29, 2006: 141-146.

27. Lapidus A, LaButti K, Foster B, Lowry S, Trong S, Goltsman E. POLISHER: An effective tool for using ultra short reads in microbial genome assembly and finishing. AGBT, Marco Island, FL, 2008.

28. Hyatt D, Chen GL, LoCascio PF, Land ML, Larimer FW, Hauser LJ. Prodigal: prokaryotic gene recognition and translation initiation site identification. BMC Bioinformatics 2010; 11:119. PubMed doi:10.1186/1471-2105-11-119

29. Pati A, Ivanova NN, Mikhailova N, Ovchinnikova G, Hooper SD, Lykidis A, Kyrpides NC. GenePRIMP: a gene prediction improvement pipeline for prokaryotic genomes. Nat Methods 2010; 7:455-457. PubMed doi:10.1038/nmeth.1457

30. Markowitz VM, Ivanova NN, Chen IMA, Chu K, Kyrpides NC. IMG ER: a system for microbial genome annotation expert review and curation. Bioinformatics 2009; 25:2271-2278. PubMed doi:10.1093/bioinformatics/btp393 